\title{
The diversification of PHIS transposon superfamily in eukaryotes
}

\author{
Min-Jin Han', Chu-Lin Xiong ${ }^{1}$, Hong-Bo Zhang ${ }^{1}$, Meng-Qiang Zhang ${ }^{1}$, Hua-Hao Zhang ${ }^{2}$ and Ze Zhang ${ }^{\text {** }}$
}

\begin{abstract}
Background: PHIS transposon superfamily belongs to DNA transposons and includes PIF/Harbinger, ISL2EU, and Spy transposon groups. These three groups have similar DDE domain-containing transposases; however, their coding capacity, species distribution, and target site duplications (TSDs) are significantly different.

Results: In this study, we systematically identified and analyzed PHIS transposons in 836 sequenced eukaryotic genomes using transposase homology search and structure approach. In total, 380 PHIS families were identified in 112 genomes and 168 of 380 families were firstly reported in this study. Besides previous identified PIF/Harbinger, ISL2EU, and Spy groups, three new types (called Pangu, Nuwal, and Nuwall) of PHIS superfamily were identified; each has its own distinctive characteristics, especially in TSDs. Pangu and Nuwall transposons are characterized by 5'-ANT-3' and 5'-C|TNA|G-3' TSDs, respectively. Both transposons are widely distributed in plants, fungi, and animals; the Nuwal transposons are characterized by 5'-CWG-3' TSDs and mainly distributed in animals.

Conclusions: Here, in total, 380 PHIS families were identified in eukaryotes. Among these 380 families, 168 were firstly reported in this study. Furthermore, three new types of PHIS superfamily were identified. Our results not only enrich the transposon diversity but also have extensive significance for improving genome sequence assembly and annotation of higher organisms.
\end{abstract}

Keywords: Transposable elements, PHIS, Diversification, Identification

\section{Background}

Transposable elements (TEs) are fragments of DNA that can move from one site to another in a genome $[1,2]$. TEs are classified into two classes (class 1 and class 2) according to their mechanism of transposition. The transposition mechanism of class 1 elements can be described as copy-and-paste mode, whereas class 2 transposons can be transposed by cut-and-paste mechanism. Recently, more and more genome sequencing revealed that TEs constitute the largest components of most eukaryotic genomes [2-13]. TEs not only have significant impact on the evolution of the host genomes and biological complexity but also are challenges for host genome sequencing, assembly, and annotation due to their repeatability. Thus, the knowledge about TEs characteristics and categories will promote the development of genomics.

\footnotetext{
* Correspondence: zezhang@cqu.edu.cn

${ }^{1}$ School of Life Sciences, Chongqing University, Chongqing 400044, China Full list of author information is available at the end of the article
}

In the past decade, many studies focused on identification, annotation, and function of TEs. So far, huge amounts of TEs have been identified and annotated. For example, 42 class 1 superfamilies and 19 class 2 superfamilies were annotated and cataloged in the RepBase database. However, the number of reported TEs could be just the tip of the iceberg. There are a larger number of TEs to be annotated due to their great diversification. For instance, 658 families were classified into unknown TEs in the silkworm; 163 unknown TE families in the maize and about $0.38 \%$ of mouse genome sequences are unknown TEs [12-14]. Thus, the work of identification and annotation of TEs is far from finished.

Recently, we have identified a new group of cut-andpaste transposons designated as Spy [15]. Spy transposons are distinct from all other groups of DNA transposons by their strong insertion preference within the AAATTT motif and the lack of target site duplications (TSDs) upon insertion. In addition, we showed that PIF/ Harbinger, ISL2EU, and Spy are evolutionarily related and share a preference for insertion into AT-rich target 
sequences [15]. For instance, the ISL2EU transposons are characterized by $5^{\prime}$-AT-3' TSDs and the PIF/Harbinger transposons by $5^{\prime}$-TWA-3' $[16,17]$. Thus, these three groups PIF/Harbinger, ISL2EU, and Spy were classified into the same superfamily that is designated as "PHIS". The PHIS transposon superfamily is high polymorphism in the target sequences, coding capacity, and conserved motifs of transposase [15]. It is common to find some distinct groups within a given superfamily. Previously, variable nucleotide composition and length of TSDs were found in some superfamilies [16-18]. However, the detailed diversification of PHIS transposon superfamily still remains unclear.

Here, we systematically identified and analyzed PHIS transposons in 836 sequenced eukaryotic genomes using transposase homology search combined with structure approach. Totally, 380 PHIS families including 212 previously reported families and 168 unpublished families were identified in this study. The 380 PHIS families are classified into six groups including three previously reported groups (PIF/Harbinger, ISL2EU, and Spy) and three new groups, called Pangu, NuwaI, and NuwaII. Each new group has its own particular characteristics, especially in TSDs.

\section{Results}

\section{The landscape of PHIS transposons in eukaryotic}

\section{genomes}

To investigate the detailed diversification and evolution of PHIS superfamily in eukaryotes, we systematically identified and analyzed the characteristics and distribution of PHIS transposons in 836 eukaryotic genomes using transposase homology search and structure approach. Finally, we identified 380 PHIS transposon families. Furthermore, each of the PHIS consensus sequence defined in this study was subject to homology search against RepBase (as of October 20, 2014) and National Center for Biotechnology Information (NCBI) nonredundant (nr) nucleotide database using Censor and BlastN program. The results of these searches showed that 168 of 380 PHIS families were not reported, and other TEs (212) had been released and cataloged in RepBase, NCBI, or published papers [15].

Based on the characteristics (TSDs, coding capacity, and secondary structure of transposase, etc.) of these 380 families, we found that 214 families belong to the PIF/Harbinger transposon group (Additional file 1: Table S1). Among the 214 families, 80 families had been previously identified and cataloged in RepBase, and 134 families were firstly identified in this study. These 214 families shared the following characteristics. (1) The TSD sequence is 5 '-TWA-3' tri-nucleotide ('W' represents A or T nucleotide) (Fig. 1). (2) Most candidate autonomous elements contain two open reading frames
(ORFs), one ORF encoding the DDE and helix-turnhelix $(\mathrm{HTH})$ motif-containing transposase and the other ORF encoding a DNA-binding protein with a Myb/ SANT domain. The potential active families of PIF/Harbinger group were defined as those including both two intact ORFs. Finally, we identified 88 potential active families in the eukaryotic genomes (Additional file 1: Table S1 and Fig. 2b). (3) The TIR (terminal inverted repeat) lengths of different PIF/Harbinger families are highly variable (5-1042 bp), but the lengths of most TIRs $(\sim 93 \%)$ are less than $60 \mathrm{bp}$, and the first nucleotide of TIRs is usually A or G (Fig. 1). (4) The average length of consensus sequences of candidate autonomous is $\sim 4124 \mathrm{bp}$. (5) These families are distributed in 75 species including plants, fungi, and animals. The abovedescribed characteristics of PIF/Harbinger transposons are consistent with previous reports $[15,16,19]$.

Meanwhile, 25 families belong to ISL2EU group. Among these 25 families, 8 families were firstly identified in this study. The others had been cataloged in RepBase (Additional file 1: Table S2). These families shared the following characteristics. (1) The TSDs are 5'-AT-3' di-nucleotide; however, there is a conserved single A nucleotide in the flank of $5^{\prime}$ terminal of TSDs and a conserved single $\mathrm{T}$ nucleotide in the flank of 3' terminal of TSDs (Additional file 2: Figure S1). Thus, we speculated that the target site sequence of ISL2EU transposons is $\mathrm{A}|\mathrm{AT}| \mathrm{T}$ (where 'l' marks the cut site), the analysis of paralogous empty sites further confirmed the target site sequence of ISL2EU. Additional file 2: Figure S2 shows the possible generation mechanism of this TSDs. (2) Most autonomous candidate transposons of ISL2EU contain two ORFs, one ORF encoding the DDE, $\mathrm{HTH}$, and THAP domain-containing transposase, the other ORF encoding a DNA-binding protein with a YqaJ exonuclease domain. Similar to a standard mentioned before, TEs with two intact ORFs are defined as the potential active transposons. Thus, 12 potential active families of ISL2EU group were identified in the eukaryotic genomes (Additional file 1: Table S2 and Fig. 2b) (3). The TIR length ranges from 6 to $259 \mathrm{bp}$, and the first two nucleotides of TIRs are usually "GG" di-nucleotide (Fig. 1). (4) The average length of consensus sequences of autonomous elements is $\sim 4840 \mathrm{bp}$. (5) These families are distributed in 14 species. All these species belong to animals.

In this study, we found 54 families that belong to the Spy transposons; however, we did not identify any new Spy transposon family. All these families have been identified in previous study, and the characteristics of Spy transposons were also shown previously [15]. Besides the above three identified PHIS groups (PIF/Harbinger, $I S L 2 E U$, and Spy), we also found three new types of PHIS transposons distinct from the previous PHIS 


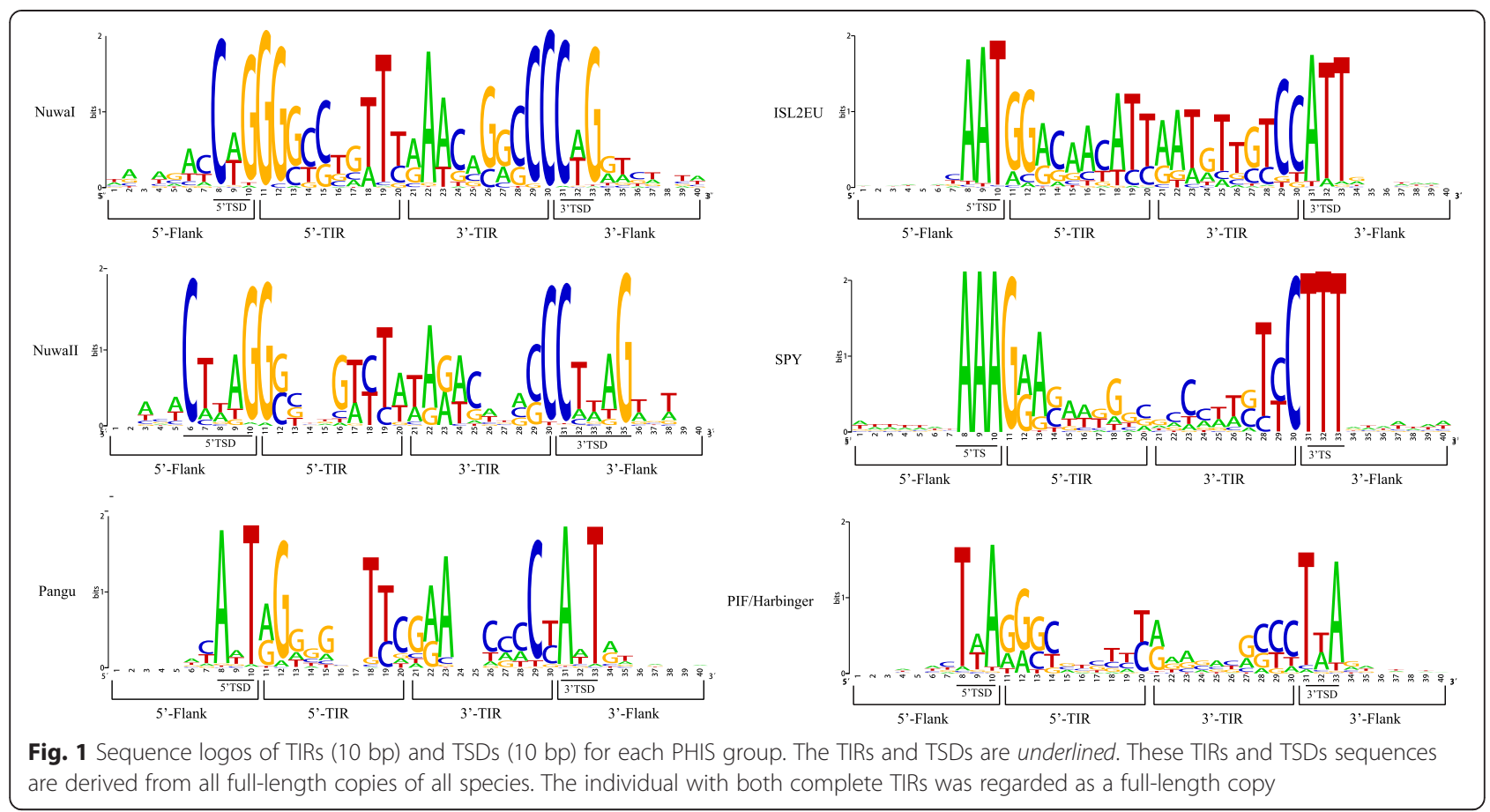

transposons in TSDs, and these new types transposons are called Pangu, NuwaI, and NuwaII, respectively.

\section{Characterization and distribution of Pangu transposons}

Thirty four Pangu families were identified in this study (Additional file 1: Table S3). The length of TIRs in these families varies from 11 to $40 \mathrm{bp}$, and the first two nucleotides of TIRs are usually "AG" and "GG" di-nucleotide (Fig. 1). The average consensus sequence length of autonomous candidates is $\sim 3487 \mathrm{bp}$. Most autonomous candidates of Pangu transposon contain two ORFs, one ORF encoding the DDE motif-containing transposase and without any other domains. Meanwhile, we did not detect any known motifs in the other ORF. Given that the potential active families should contain the two intact ORFs, we identified two potential active families of Pangu group in the eukaryotic genomes (Additional file 1: Table S3 and Fig. 2b). Secondary structure prediction of Pangu DDE-containing transposases suggests that the first $\mathrm{D}$ is located between two beta-sheets, the second D is located between a beta-sheet and an alphahelix, and the last $\mathrm{E}$ is present within an alpha-helix (Fig. 3). This result is consistent with the eukaryotic PIF/ Harbinger and ISL2EU transposons [15]. The results of paralogous empty site confirmed that the TSDs of these families are 5'-ANT-3' ('N' represents A, T, C, or G nucleotide) (Fig. 3). This characteristic of TSDs is significantly different from eukaryotic PIF/Harbinger, ISL2EU, and Spy transposons but consistent with the bacterial IS5 transposons. Thus, both Pangu and IS5 transposons could belong to the same group or were derived from the same ancient element.

These 34 Pangu transposons are distributed in 15 eukaryotic genomes. These species include two coleopterans, one dipteran, one arachnidan, one molluscan, one hydrozoan, one anthozoan, two ascomycetes, three basidiomycetes, one heterokontophyta, and two algae (Fig. 2). And these species are widely distributed in plants, fungi, and animals. Thus, the Pangu transposons could be ancient elements in the eukaryotic genomes. To estimate the abundance of Pangu transposons in the eukaryotic genomes, the consensus sequence of each family of Pangu was used as query in BlastN $\left(e<10^{-5}\right)$ search against the corresponding genome. A copy for the same family was defined by $e$ value less than $e^{-5}$, length larger than $50 \mathrm{bp}$, and nucleotide identity larger than $80 \%$. Finally, we identified 3270 copies of Pangu group in the eukaryotic genomes (Additional file 1: Table S3, Additional file 3: Table S4, and Fig. 2c).

\section{Characterization and distribution of Nuwal transposons}

Twenty-three NuwaI families were identified in this study (Additional file 1: Table S5). The results of paralogous empty site confirmed that the TSDs of these families are 5'-CWG-3' ('W' represents A or T nucleotide) (Fig. 4). This characteristic is significantly different from previously the identified PIF/Harbinger, ISL2EU, and Spy transposons (AT-rich TSDs). Most autonomous candidates of NuwaI transposons contain two ORFs, one ORF encoding the DDE motif-containing transposase and 


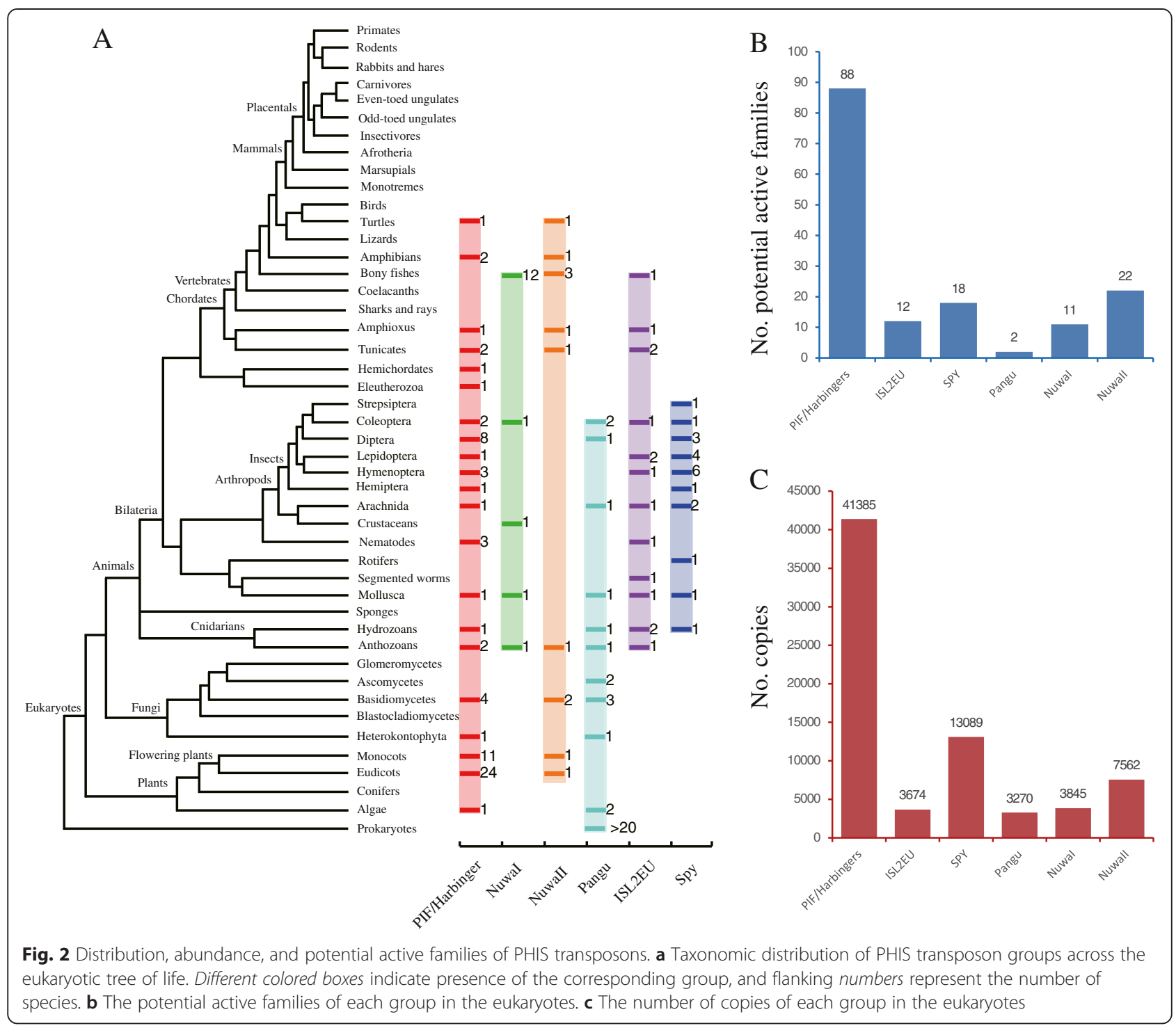

without any other domain, the other ORF encoding a DNA-binding protein with a Myb/SANT domain. We identified 11 potential active families in the eukaryotic genomes because these TEs contain the two intact ORFs (Additional file 1: Table S5 and Fig. 2b). The secondary structure of NuwaI transposase is very similar to the PIF/Harbinger, ISL2EU, and Pangu transposases. For instance, the first D is located between two beta-sheets, the second D is typically between a beta-sheet and an alpha-helix, and the last E occurs within an alpha-helix (Fig. 4). The TIR lengths of NuwaI families range from 12 to $61 \mathrm{bp}$, and the first three nucleotides of TIRs are usually 'GGG' tri-nucleotide (Fig. 1). The average length of consensus sequences of autonomous candidates is $\sim 462 \mathrm{bp}$. These NuwaI transposons are distributed in 16 animal genomes. These species include 12 bony fish, 1 coleopteran, 1 crustacean, 1 molluscan, and 1 anthozoan (Fig. 2a). However, these species are distributed only in the kingdom of animals. Thus, the NuwaI transposons could be relatively younger elements in the eukaryotes. Finally, 3845 copies of NuwaI group were identified in the eukaryotic genomes. The genomic abundance and copy number of each NuwaI family in each species were shown in Fig. 2c, Additional file 1: Table S5, and Additional file 3: Table S6.

\section{Characterization and distribution of Nuwall transposons}

There are 30 out of 380 families which belong to the NuwaII families (Additional file 1: Table S7). According to the paralogous empty site, we cannot judge that the TSDs of NuwaII group are 3 bp (TNA) or 5 bp (CTNAG) (Fig. 5). However, most PHIS elements are typically associated with 3-bp TSD. Thus, the TSDs of NuwaII elements are most likely 3-bp TSDs. Meanwhile, 


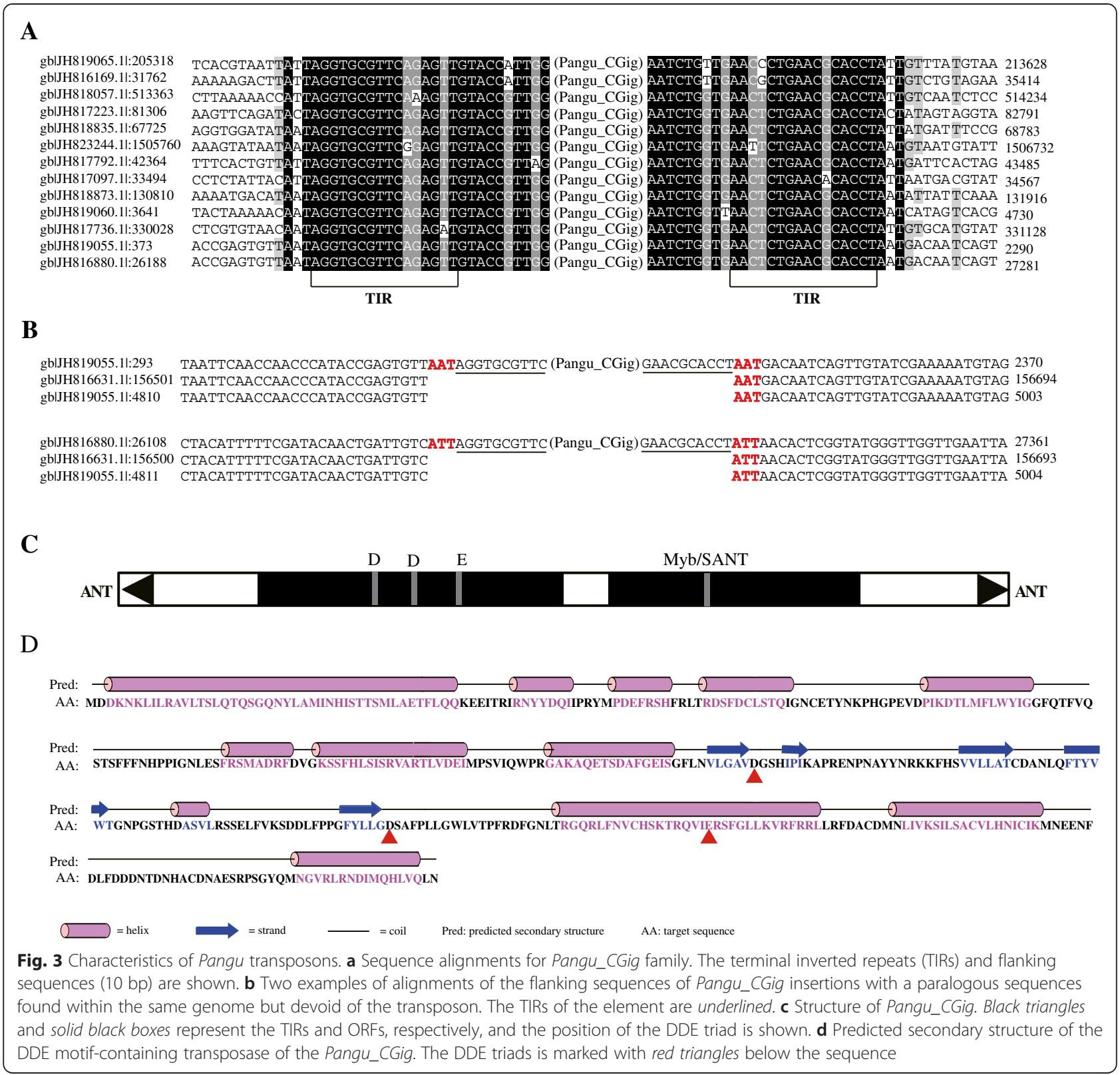

there is a conserved single $C$ nucleotide in the flank of 5 ' terminal of TSDs and a conserved single $G$ nucleotide in the flank of 3' terminal of TSDs. Thus, the target of NuwaII is preferentially C|TNA|G (' $N$ ' represents A, T, $\mathrm{C}$, or $\mathrm{G}$ nucleotide, '|' represents the cut site).

The transposase of NuwaII is very similar to that of NuwaI in the coding capacity, conserved motifs, and second enzyme structure. For instance, the most autonomous elements of NuwaII transposons contain two ORFs, one ORF encoding the DDE motif-containing transposase (Additional file 2: Figure S3), and the other ORF encoding a Myb/SANT domain-containing protein. Twenty-two potential active NuwaII families with the two intact ORFs were identified in the eukaryotic genomes (Additional file 1: Table S7 and Fig. 2b). In the secondary structure of NuwaII transposase, the first D is located between two beta-sheets, the second D is typically between a beta-sheet and an alpha-helix, and the last E occurs within an alpha-helix (Fig. 5). The average length of consensus sequences of autonomous candidates is $\sim 4685 \mathrm{bp}$; TIRs length of each family ranges from 13 to $46 \mathrm{bp}$, and the first two nucleotides of most TIRs are conserved GG. These NuwalI transposons are distributed in 12 species, including 1 turtle, 1 amphibian, 3 bony fishes, 1 amphioxus, 1 tunicate, 1 anthozoan, 2 basidiomycetes, 1 monocot, and 1 eudicot (Fig. 2a). Meanwhile, these species are also distributed in the kingdoms of plants, fungi, and animals. Thus, the 


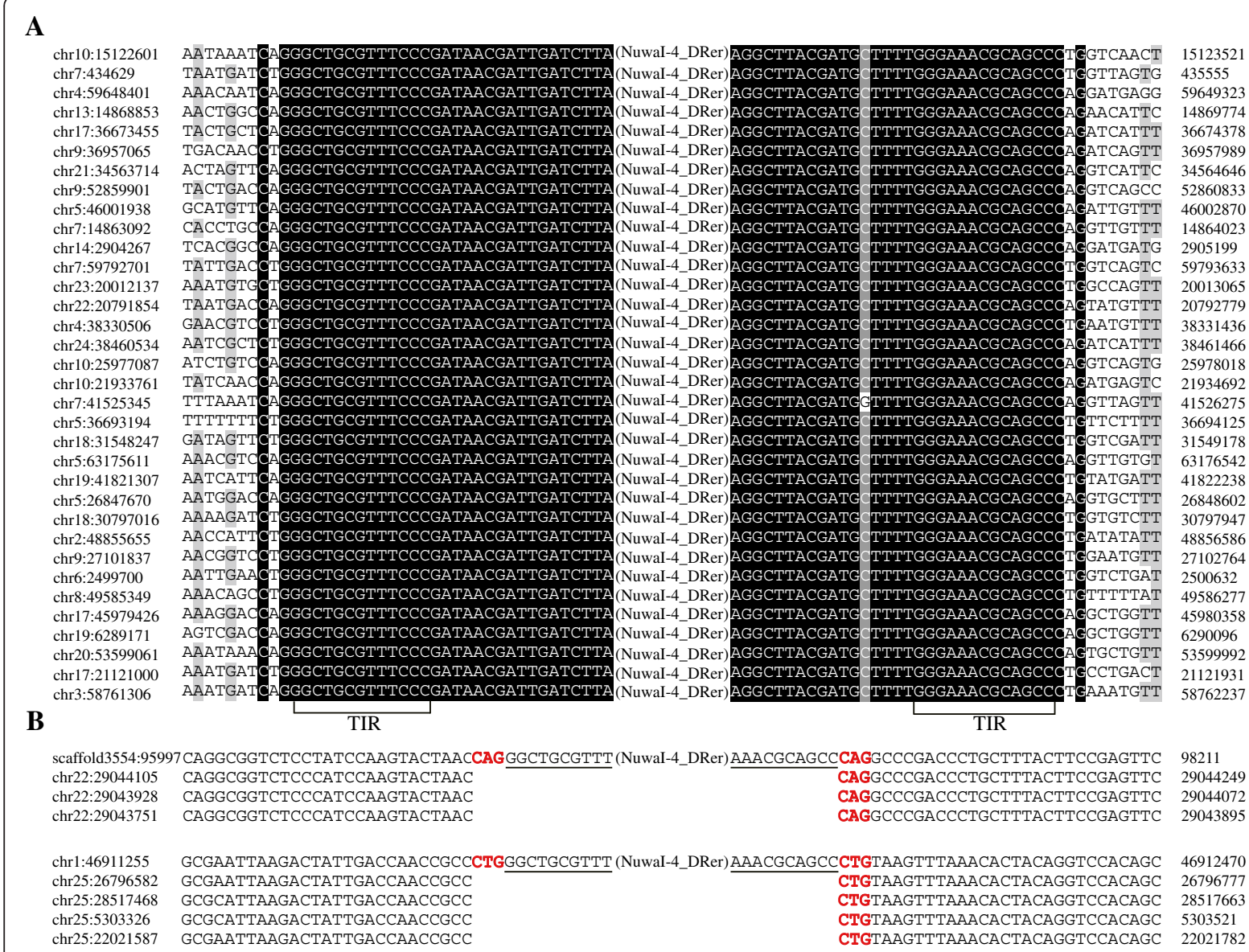

C

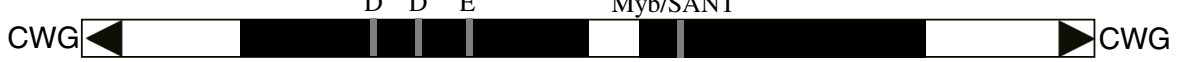

D

Pred:

Pred: Pred: Pred:

AA: QQVIDTFF

$\longrightarrow=$ helix $\longrightarrow=$ strand $\longrightarrow=$ coil $\quad$ Pred: predicted secondary structure AA: target sequence

Fig. 4 Characteristics of Nuwal transposons. a Sequence alignments for Nuwal-4_DRer family. The terminal inverted repeats (TIRs) and flanking sequences (10 bp) are shown. b Two examples of alignments of the flanking sequences of Nuwal-4_DRer insertions with a paralogous sequences found within the same genome but devoid of the transposon. The TIRs of the element are underlined. c Structure of Nuwal-4_DRer. Black triangles and solid black boxes represent the TIRs and ORFs, respectively, and the position of the DDE triad is shown. $\mathbf{d}$ Predicted secondary structure of the DDE motif-containing transposase of the Nuwal-4_DRer. The DDE triads is marked with red triangles below the sequence

NuwaII transposons could be also relatively old elements. Finally, we found 7564 copies of NuwaII group. The genomic abundance and copy number of each NuwaII family in each species are shown in Fig. 2c and Additional file 3: Table S8.

\section{Evolutionary relationships of PHIS transposons}

To investigate the evolutionary relationships of six PHIS transposon groups (PIF/Harbinger, ISL2EU, Spy, Pangu, NuwaI, and NuwaII), the core catalytic DDE domain of 16 representative transposases (include intact DDE 


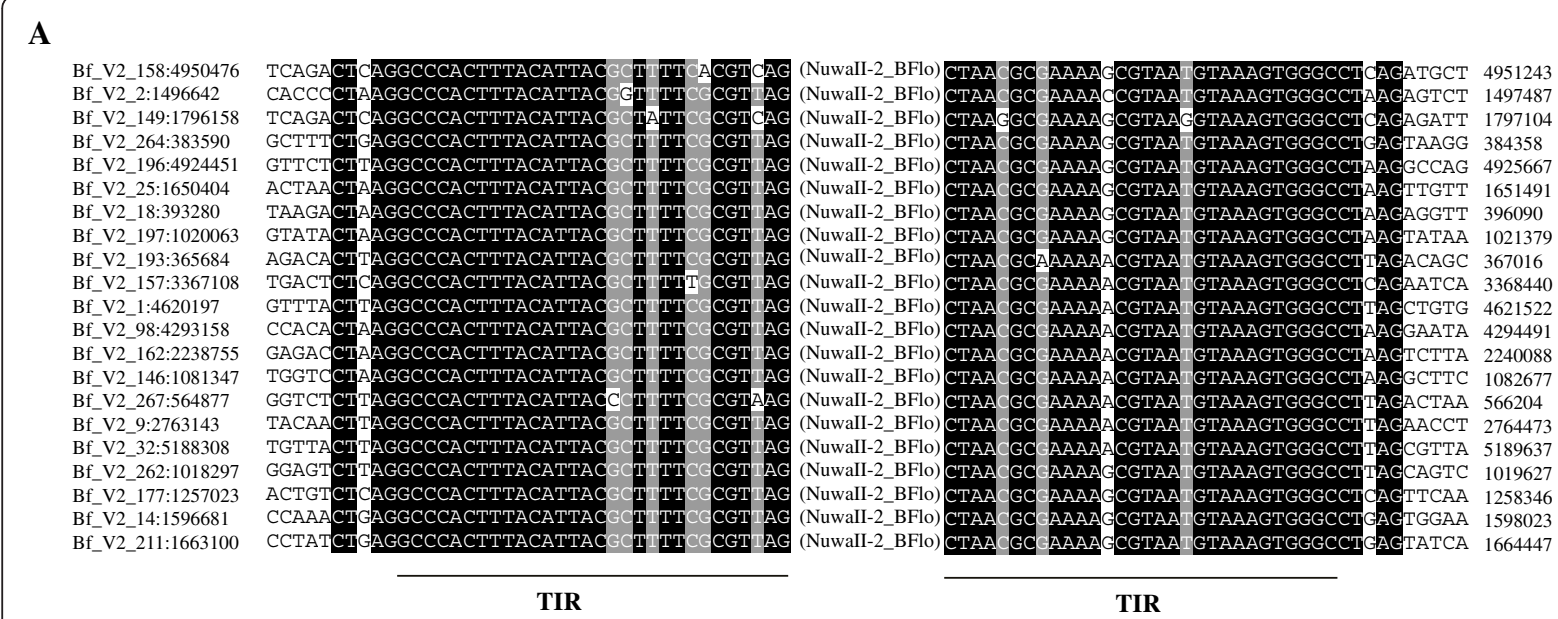

B

Bf_V2 157:3367028 TGTATATCAACCCCCTTCTGTGATTCTGAGGCCCACTTTA (NuwaII-2_BFlo) TAAAGTGGGCCTGAGAGTCAGGTATTAGCATTTCACTACA 3368520 Bf_V2_157:3366540 TGTATAGCAACCTCCTCCTCTGATT - CTGAGAGTCAGGTATTAGCATTATACTACA 3366734

Bf_V2_157:3367028 TGTATATCAACCCCCTTCTGTGATTCTGAGGCCCACTTTA (NuwaII-2_BFlo) TAAAGTGGGCCTGAGAGTCAGGTATTAGCATTTCACTACA 3368520 Bf_V2_157:3366540 TGTATAGCAACCTCCTCCTCTGATTC CATAGCAGGTATHCCATSACA 3366734

C

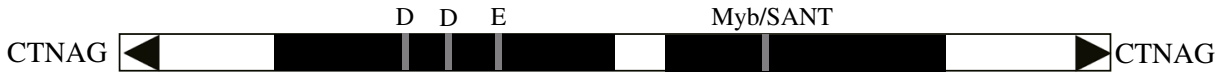

$\mathrm{D}$

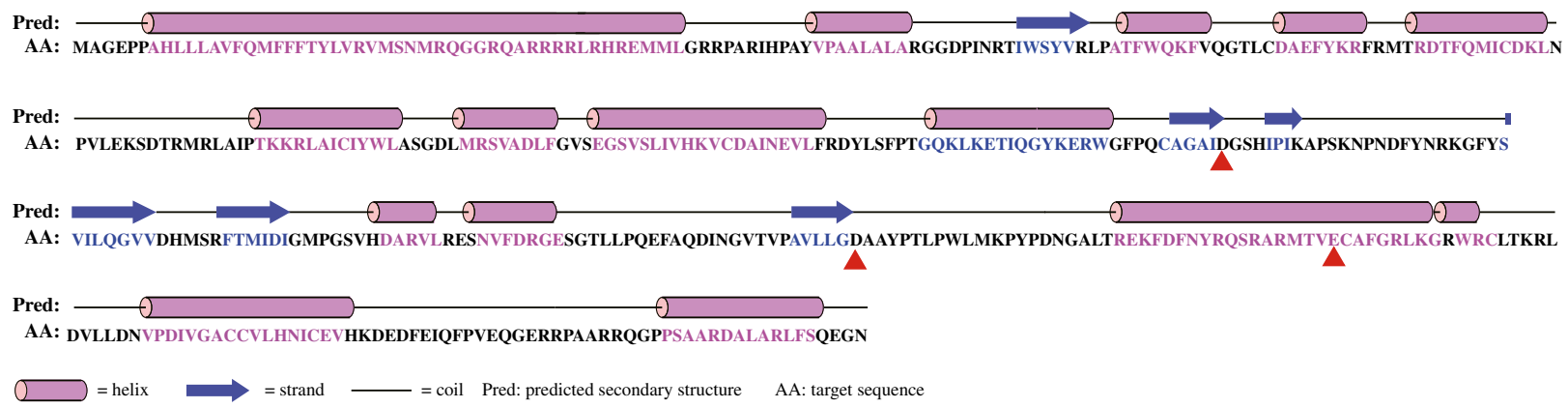

Fig. 5 Characteristics of Nuwall transposons. a Sequence alignments for Nuwall-2_BFlo family. The terminal inverted repeats (TIRs) and flanking sequences (10 bp) are shown. $\mathbf{b}$ Examples of alignments of the flanking sequences of Nuwall-2_BFlo insertions with a paralogous sequences found within the same genome but devoid of the transposon. The TIRs of the element are underlined. $\mathbf{c}$ Structure of Nuwall-2_BFlo. Black triangles and solid black boxes represent the TIRs and ORFs, respectively, and the position of the DDE triad is shown. $\mathbf{d}$ Predicted secondary structure of the DDE motif-containing transposase of the Nuwall-2_BFlo. The DDE triads is marked with red triangles below the sequence

domain) of Pangu, 12 NuwaI, 21 NuwaII, 33 PIF/ Harbinger, 18 ISL2EU, 11 Spy, and 11 bacterial IS5 were used to perform a Bayesian phylogenetic analysis. The resulting tree (Fig. 6) showed that the eukaryotic PHIS transposases formed five distinct highly supported monophyletic clades beside the individual clade of bacterial IS5 transposons. In the phylogenetic tree, Pangu, PIF/Harbinger, ISL2EU, and $S P Y$ transposons formed four separate clades. Meanwhile, NuwaI and NuwaII transposons formed a single clade in the phylogenetic tree.

\section{Discussion}

Identification and characterization of PHIS transposons

Previous study suggested that the PHIS is a DNA transposon superfamily with a great diversity in the eukaryotic genomes [15]. However, the detailed diversification and evolution of PHIS superfamily are still unknown. In this study, we systematically identified PHIS transposons in the eukaryotic genomes. A total of 380 families of PHIS superfamily were identified in 112 sequenced eukaryotic genomes. These families were classified into six groups based on the characteristic of each family's TSDs. Among 


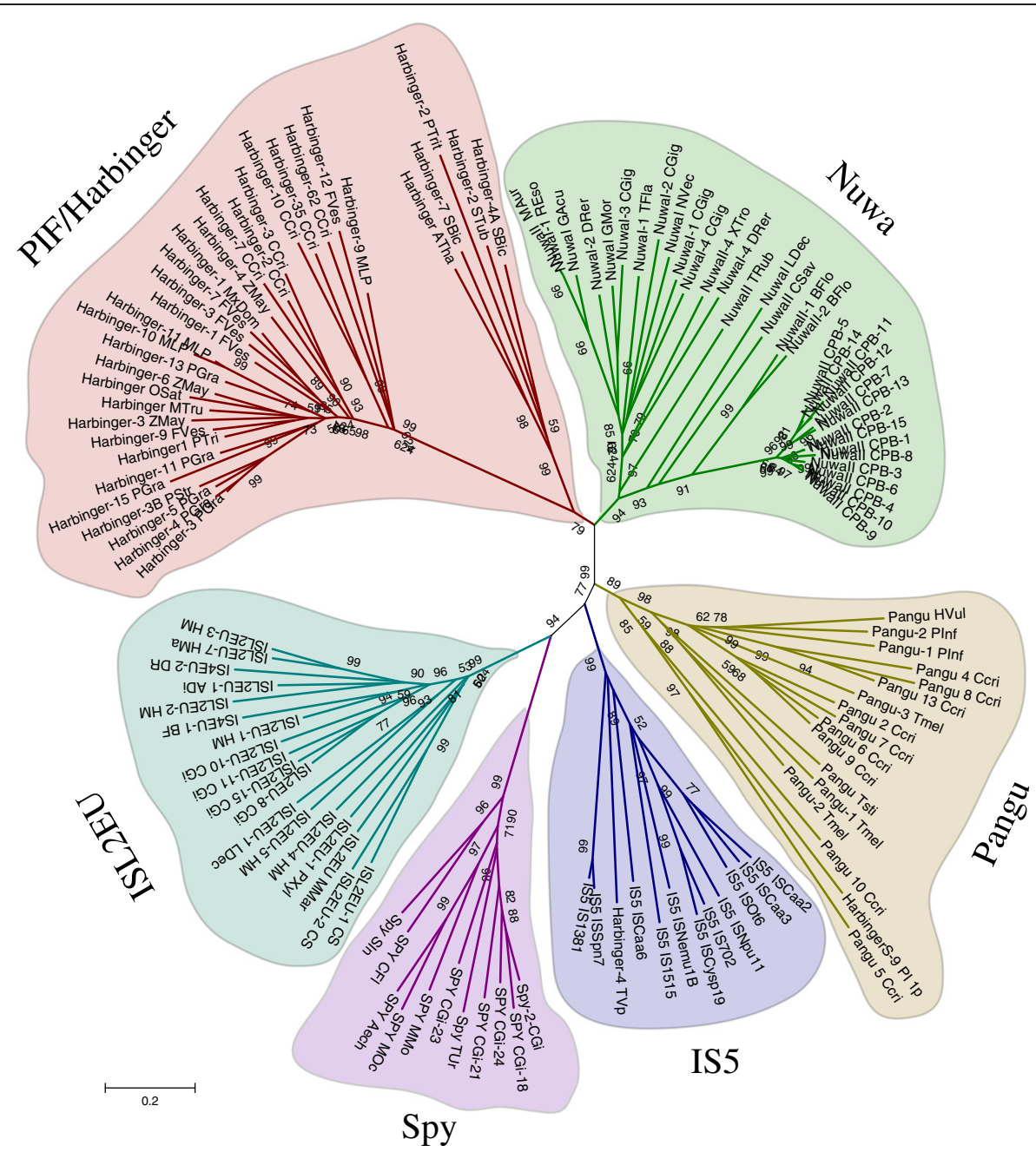

Fig. 6 Phylogenetic tree based on DDE domain sequences of PHIS superfamily

these groups, three (PIF/Harbinger, ISL2EU, and Spy) have been reported in the previous studies [15, 20, 21]. Beside the above three groups, we found three new transposon groups, called Pangu, NuwaI, and NuwaII.

These types shared similar transposases with DDE motif. However, each group has unique TSDs distinguished from others (Additional file 2: Figure S2). According to the criteria of previous TE classification [16], the transposases can be aligned over their entire catalytic regions ( $e$ value less than $e^{-4}$ ), then they belong to the same superfamily. The same group of a superfamily was defined by the same TSD composition. In addition, previous studies showed that variable length or composition of TSDs have been identified in some superfamilies, such as $8-$ 9 bp TSDs in Merlin superfamily, 5-8 bp in hAT, 2-4 bp in $C M C$, and $4-5$ bp in Ginger [16, 22, 23]. Thus, it may be better to define Spy, PIF/Harbinger, and ISL2EU and Pangu, NuwaI, and NuwaII as different groups (at the same level) of the same superfamily (PHIS).
To estimate the abundance of each group in the eukaryotic genomes, the consensus sequence of each family of each group was used as a query in BlastN $\left(e<10^{-5}\right)$ search against corresponding genome. Finally, we found that the abundances of these transposon groups varied in the eukaryotic genomes. For instance, there were 41,385 copies of PIF/Harbinger group, 3647 copies of ISL2EU, 13,089 copies of SPY, 3270 copies of Pangu, 3845 copies of NuwaI, and 7562 copies of NuwaII in the eukaryotic genomes (Additional files 1 and 3: Table S1-S8 and Fig. 2c). However, it should be noted that PHIS transposons were investigated using transposase homology search. Thus, some nonautonomous PHIS transposons (such as MITEs) might be missed in this study. In addition, we found that the number of potential active families varied. For example, there were 88 potential active families of PIF/ harbinger, 12 families of ISL2EU, 18 families of SPY, 2 families of Pangu, 11 families of NuwaI, and 22 families of NuwaII in the eukaryotes (Fig. 2b). Furthermore, the 
abundance of each group was significantly positively correlated with the number of potential active families (Pearson's product-moment correlation, $r=0.9816605$, $P=0.0005)$. This phenomenon is easy to understand, and the more potential active families will have more copies for a group of PHIS transposon superfamily.

Most groups of PHIS superfamily include two ORFs, one coding for transposase containing DDE motif and the other ORF encoding a DNA-binding protein. However, $S P Y$ transposons include only one transposase containing DDE motif [15]. In addition, the additional ORFs of the four groups (including Pangu, PIF/Harbinger, NuwaI, and NuwaII) encode a protein with Myb/SANT domain except that of the ISL2EU transposon that encodes a protein with the Yqaj domain. At present, the functions of the additional ORFs are still unknown, and whether these ORFs are related to the transposition mechanisms also remains unclear [24]. This question could be answered using biochemical studies in the future.

The results of species distribution of PHIS transposons showed that the PHIS elements are completely absent in mammals, birds, sponges, sharks, and coelacanths. This is consistent with a previous study [16]. In addition, it is interesting to see that in some lineages, there is only one of the six groups of PHIS superfamily or only one of the six groups is absent. To our knowledge, the above results could be caused by two reasons. First, some PHIS transposons were lost or degenerated in some species by drift or selection in their original lineages. Second, some species gain different families from other species through horizontal transfer (HT). In addition, almost all of the DNA transposons have the ability of HT, and more and more HT of DNA transposons have been reported in the eukaryotic genomes [25-29]. Furthermore, previous studies suggested that PIF/Harbinger experienced HT events between Drosophila species [30]. However, HT of PHIS transposons remains to be studied in the future.

\section{Evolutionary relationships of PHIS transposons}

The result of phylogenetic analysis showed that Pangu elements formed a single clade and were adjacent to IS5 group in the phylogenetic tree. In addition, both Pangu and IS5 transposons shared the same target site sequence ( $5^{\prime}$-ANT-3'). Furthermore, Pangu elements were widely distributed in plants, fungi, and animals. Thus, we proposed that Pangu is a relatively old PHIS group in the eukaryotic genomes.

Meanwhile, NuwaI and Nuwall transposons formed a single clade in the phylogenetic tree, and they shared the same coding capacity (encoding two ORFs) and the conserved domains (DDE motif and Myb/SANT domain). However, the TSDs of NuwaI are significantly different from the NuwaII transposons. NuwaI and NuwaII transposons should belong to two different groups of PHIS superfamily. Nevertheless, these two types might diverge recently. Thus, the two types cannot be distinguished from each other in the phylogenetic tree.

HarbingerS-9_PI and Harbinger-4_TV had been released as PIF/Harbinger families cataloged in RepBase. However, our phylogenetic analysis indicated that HarbingerS-9_PI was grouped into the clade of Pangu group. Meanwhile, Harbinger-4_TV was grouped into the IS5 clade (Fig. 6). However, we could not find distinct target site duplications (TSDs) in the flank of HarbingerS-9_PI and Harbinger-4_TV families. Right now, we cannot judge if both families should belong to which group of PHIS superfamily.

\section{Conclusions}

In the present study, 380 PHIS transposon families were identified in 112 of 836 sequenced eukaryotic genomes using transposase homology search and structure approach. Among these families, 168 families are firstly identified in this study. We systematically analyzed their characteristics including TSDs, TIRs, coding capacity, conserved transposase domain and species distribution, etc. The phylogenetic analysis based on the core catalytic DDE domain of these identified transposases showed that these PHIS transposon families were divided into five clusters including three previous reported clusters (PIF/Harbinger, ISL2EU, and Spy) and two new clusters (Pangu and Nuwa). Nuwa cluster includes two groups called NuwaI and NuwaII. Furthermore, each new group has its own distinctive characteristics, especially in target site sequences. For instance, the Pangu transposons are characterized by $5^{\prime}$-ANT-3' TSDs, the NuwaI transposons by $5^{\prime}-\mathrm{CWG}-3^{\prime}$, and the NuwaII transposons by $5^{\prime}-$ C|TNA|G-3'. Our results reveal the diversification and evolution of PHIS transposons in the eukaryotic genomes and imply that further study on the generation mechanism of varied target sequences of PHIS superfamily will promote the development of new transgenic vectors.

\section{Methods}

\section{Identification of PHIS superfamily}

Eukaryotic genomes including animals (295 species), plants (105 species), fungi (315 species), and protists (121 species) were downloaded from NCBI (http:// www.ncbi.nlm.nih.gov/) (as of January 16, 2014), and the information of each species is listed in Additional file 3: Table S9. All published autonomous PHIS elements were downloaded from RepBase (v19.07) [31]. PHIS elements of eukaryotic genomes were identified using the transposase homology search that includes three steps (Additional file 2: Figure S4): (1) the transposase sequences of published 
PHIS elements were used as a query to do TblastN and TESeeker searches against each genome [32], where a hit with $e$ value less than $10^{-4}$ was considered as candidate PHIS sequence; (2) each candidate PHIS nucleotide sequence was used as a query to BlastN search $\left(e\right.$ value $<e^{-5}$, sequence length $>50 \mathrm{bp}$, and nucleotide identity $>80 \%$ ) against the corresponding genome; (3) the sequences of each cluster were extended in both directions using a Perl script and aligned using MUltiple Sequence Comparison by Log-Expectation (MUSCLE) [33], then the boundaries of each cluster were manually defined.

\section{Characterization and phylogenetic analysis of PHIS superfamily}

To estimate the abundance of each PHIS family in the corresponding genome, the consensus sequence of each family was used as a query in BlastN search against the corresponding genome. Finally, the sequences with the $e$ value less than $e^{-5}$, length larger than $50 \mathrm{bp}$, and a minimum nucleotide identity of $80 \%$ were classified as members of the same family. Transposase coding sequences, transposase domains, secondary structures of representative transposases, and the paralogous empty sites were analyzed as described previously [15]. Sequence logos of TIRs and TSDs were created by WebLogo (http://weblogo.berkeley.edu/logo.cgi) [34]. Multiple sequences alignments were performed using MUSCLE software with default parameters. The phylogenetic tree was constructed based on the DDE domains of transposases using MrBayes software (v3.1.2) [35] with the Blosum model and other parameters with default. The Blosum model was estimated by protest- 3.2 software [36]. Meanwhile 3,000,000 generations of Bayesian inference were performed.

\section{Additional files}

Additional file 1: Table S1. Distribution and characteristics of all identified PIF/Harbinger transposons. Table S2. Distribution and characteristics of all identified ISL2EU transposons. Table S3. Distribution and characteristics of all identified Pangu transposons. Table S5. Distribution and characteristics of all identified Nuwal transposons. Table S7. Distribution and characteristics of all identified Nuwall transposons. Table S9. The eukaryotes used in this study.

Additional file 2: Figure S1. (A) Sequence alignments for ISL2EU-2_Pxyl family. The terminal inverted repeats (TIRs) and flanking sequences (10 bp) are shown. (B) An example of alignments of the flanking sequences of ISL2EU-2_Pxyl insertion with a paralogous sequences found within the same genome but devoid of the transposon. The TIRs of the element are underlined. Figure S2. Speculated transposition mechanism of each PHIS groups. Figure S3. The alignment of DDE domain of Pangu and Nuwa groups after redundancy elimination. Distances between the conserved blocks are indicated in the number of amino acid residues. Conserved residues within each superfamily are highlighted in the same color. The DDE triad identified here is marked with asterisks below alignments. Figure S4. Pipeline for PHIS transposons identification. Where a hit with $e$ value less than $10^{-4}$ was considered as a homology sequence. The ones with an $e$ value less than $e^{-5}$, sequence length larger than $50 \mathrm{bp}$, and nucleotide sequence identity larger than $80 \%$ were classified as member of the same family. Target site duplications (TSDs) were identified using the paralogous empty sites.

Additional file 3: Table S4. Positions of Pangu transposons in the corresponding genome. Table S6. Positions of Nuwal transposons in the corresponding genome. Table S8. Positions of Nuwall transposons in the corresponding genome.

\section{Abbreviations}

MITEs: Miniature inverted-repeat transposable elements; TEs: Transposable elements; TIRs: Terminal inverted repeats; TSDs: Target site duplications.

\section{Competing interests}

The authors declare that they have no competing interests.

\section{Authors' contributions}

$\mathrm{MJH}$ designed the study, carried out the analyses, and drafted the manuscript. CLX, HBZ, MQZ, and HHZ did the data analyses and revised the manuscript. ZZ designed the study, supervised the study, and revised the manuscript. All authors read and approved the final manuscript.

\section{Acknowledgements}

This work was supported by the National Natural Science Foundation of China (No. 31471197 to ZZ and No. 31401106 to MJH), Postdoctoral Science Foundation of Chongqing (No. Xm2014080 to MJH), and Chongqing Graduate Student Research Innovation Project (CYB14041).

\section{Author details}

'School of Life Sciences, Chongqing University, Chongqing 400044, China. ${ }^{2}$ College of Pharmacy and Life Science, Jiujiang University, Jiujiang 332000 , China.

Received: 13 April 2015 Accepted: 17 June 2015

Published online: 24 June 2015

\section{References}

1. Feschotte C, Jiang N, Wessler SR. Plant transposable elements: where genetics meets genomics. Nat Rev Genet. 2002;3:329-41.

2. Finnegan DJ. Eukaryotic transposable elements and genome evolution. Trends Genet. 1989;5:103-7.

3. de Koning AP, Gu W, Castoe TA, Batzer MA, Pollock DD. Repetitive elements may comprise over two-thirds of the human genome. PLoS Genet. 2011;7:e1002384

4. Holt RA, Subramanian GM, Halpern A, Sutton GG, Charlab R, Nusskern DR, et al. The genome sequence of the malaria mosquito Anopheles gambiae. Science. 2002;298:129-49.

5. Kapitonov W, Jurka J. Molecular paleontology of transposable elements in the Drosophila melanogaster genome. Proc Natl Acad Sci U S A. 2003;100:6569-74.

6. Kidwell MG. Transposable elements and the evolution of genome size in eukaryotes. Genetica. 2002;115:49-63.

7. Lander ES, Linton LM, Birren B, Nusbaum C, Zody MC, Baldwin J, et al. Initial sequencing and analysis of the human genome. Nature. 2001;409:860-921.

8. Meyers BC, Tingey SV, Morgante M. Abundance, distribution, and transcriptional activity of repetitive elements in the maize genome. Genome Res. 2001;11:1660-76.

9. Nene V, Wortman JR, Lawson D, Haas B, Kodira C, Tu ZJ, et al. Genome sequence of Aedes aegypti, a major arbovirus vector. Science. 2007:316:1718-23.

10. Sanmiguel $P$, Bennetzen $J$ L. Evidence that a recent increase in maize genome size was caused by the massive amplification of intergene retrotransposons. Ann Bot. 1998;81:37-44.

11. Vicient CM, Suoniemi A, Anamthawat-Jónsson K, Tanskanen J, Beharav A Nevo E, et al. Retrotransposon BARE-1 and its role in genome evolution in the genus hordeum. Plant Cell. 1999;11:1769-84.

12. Waterston RH, Lindblad-Toh K, Birney E, Rogers J, Abril JF, Agarwal P, et al. Initial sequencing and comparative analysis of the mouse genome. Nature. 2002;420:520-62 
13. Xu HE, Zhang HH, Xia T, Han MJ, Shen YH, Zhang Z. BmTEdb: a collective database of transposable elements in the silkworm genome. Database (Oxford). 2013;2013:bat055

14. Schnable PS, Ware D, Fulton RS, Stein JC, Wei F, Pasternak S, et al. The B73 maize genome: complexity, diversity, and dynamics. Science. 2009:326:1112-5.

15. Han MJ, Xu HE, Zhang HH, Feschotte C, Zhang Z. Spy: a new group of eukaryotic DNA transposons without target site duplications. Gonome Bio Evol. 2014;6:1748-57.

16. Yuan YW, Wessler SR. The catalytic domain of all eukaryotic cut-and-paste transposase superfamilies. Proc Natl Acad Sci U S A. 2011;108:7884-9.

17. Bao W, Jurka MG, Kapitonov W, Jurka J. New superfamilies of eukaryotic DNA transposons and their internal divisions. Mol Biol Evol. 2009;26(5):983-93.

18. Kapitonov W, Jurka J. A universal classification of eukaryotic transposable elements implemented in Repbase. Nat Rev Genet. 2008:9:411-2.

19. Zhang X, Feschotte C, Zhang Q, Jiang N, Eggleston WB, Wessler SR. P instability factor: an active maize transposon system associated with the amplification of Tourist-like MITEs and a new superfamily of transposases. Proc Natl Acad Sci U S A. 2001;98:12572-7.

20. Kapitonov W, Jurka J. Molecular paleontology of transposable elements from Arabidopsis thaliana. Genetica. 1999;107:27-37.

21. Walker EL, Eggleston WB, Demopulos D, Kermicle J, Dellaporta SL. Insertions of a novel class of transposable elements with a strong target site preference at the $r$ locus of maize. Genetics. 1997:146:681-93.

22. Feschotte C. Merlin, a new superfamily of DNA transposons identified in diverse animal genomes and related to bacterial IS1016 insertion sequences. Mol Biol Evol. 2004;21:1769-80.

23. Bao W, Kapitonov W, Jurka J. Ginger DNA transposons in eukaryotes and their evolutionary relationships with long terminal repeat retrotransposons Mob DNA. 2010;1:3.

24. Jiang N, Bao Z, Zhang X, Hirochika H, Eddy SR, McCouch SR, et al. An active DNA transposon family in rice. Nature. 2003;421:163-7.

25. Schaack S, Gilbert C, Feschotte C. Promiscuous DNA: horizontal transfer of transposable elements and why it matters for eukaryotic evolution. Trends Ecol Evol. 2010;25:537-46.

26. Daniels SB, Peterson KR, Strausbaugh LD, Kidwell MG, Chovnick A. Evidence for horizontal transmission of the $P$ transposable element between Drosophila species. Genetics. 1990;124:339-55.

27. Maruyama K, Hartl DL. Evidence for interspecific transfer of the transposable element mariner between Drosophila and Zaprionus. J Mol Evol. 1991;33:514-24.

28. Zhang $\mathrm{HH}, \mathrm{Xu} H \mathrm{HE}$, Shen $\mathrm{YH}$, Han MJ, Zhang Z. The origin and evolution of six miniature inverted-repeat transposable elements in Bombyx mori and Rhodnius prolixus. Genome Biol Evol. 2013:5:2020-31.

29. Gilbert C, Hernandez SS, Flores-Benabib J, Smith EN, Feschotte C. Rampant horizontal transfer of SPIN transposons in squamate reptiles. Mol Biol Evol. 2012;29:503-15.

30. Casola C, Lawing AM, Betrán E, Feschotte C. PIF-like transposons are common in drosophila and have been repeatedly domesticated to generate new host genes. Mol Biol Evol. 2007;24:1872-88.

31. Jurka J, Kapitonov W, Pavlicek A, Klonowski P, Kohany O, Walichiewicz J. Repbase Update, a database of eukaryotic repetitive elements. Cytogenet Genome Res. 2005;110:462-7.

32. Kennedy RC, Unger MF, Christley S, Collins FH, Madey GR. An automated homology-based approach for identifying transposable elements. BMC Bioinformatics. 2011;12:130

33. Edgar RC. MUSCLE: multiple sequence alignment with high accuracy and high throughput. Nucleic Acids Res. 2004;32:1792-7.

34. Schneider TD, Stephens RM. Sequence logos: a new way to display consensus sequences. Nucleic Acids Res. 1990;18:6097-100.

35. Ronquist F, Huelsenbeck JP. MrBayes 3: Bayesian phylogenetic inference under mixed models. Bioinformatics. 2003:19:1572-4

36. Darriba D, Taboada GL, Doallo R, Posada D. ProtTest 3: fast selection of best-fit models of protein evolution. Bioinformatics. 2011:27:1164-5.

\section{Submit your next manuscript to BioMed Central and take full advantage of:}

- Convenient online submission

- Thorough peer review

- No space constraints or color figure charges

- Immediate publication on acceptance

- Inclusion in PubMed, CAS, Scopus and Google Scholar

- Research which is freely available for redistribution

Submit your manuscript at www.biomedcentral.com/submit 